EMISSION LINE GALAXIES AT $1<z<1.5$

K. GLAZEBROOK

Anglo-Australian Observatory, P.O. Box 296,

Epping, NSW 2121, Australia

R.G. ABRAHAM

Institute of Astronomy, Madingley Road, Cambridge CB3 OHA, United Kingdom

AND

\title{
C.A. BLAKE
}

Department of Physics, Astrophysics, 1 Keble Road, Oxford OX1 3NP, United Kingdom

\section{Why study emission line galaxies at high redshift?}

In this paper we wish to introduce the first results on two new projects aimed at detecting emission lines in galaxies at $z>1$. There are two primary motivations for doing this: Firstly to try and measure the cosmic starformation rate at these redshifts. The combination of $z<1$ redshift surveys and the discovery of the $z \sim 3$ Hubble Deep Field ultraviolet dropout objects has led to a 'first draft' history of the cosmic SFR (Fig. 1). These results are based on UV continuum fluxes, it is highly desirably to confirm these studies with line diagnostics and extend the work to the redshift of the inferred peak $(z \sim 1.5)$.

Secondly like the drunk looking for his keys under the lamp-post one reason for looking for emission lines galaxies is that the line/continuum contrast renders them easier to find at high- $z$ ! Since we don't yet have any significant samples of normal field galaxies in the $z \sim 1.5-2$ regime to study (primarily because the optical lines are redshifted in to the high-background near-infrared and the strong UV lines have yet to make an appearance in the optical) this is not just a throwaway point. Also for some studies, such as the cosmic SFR, samples selected down to a constant line luminosity can be regarded as complete. 


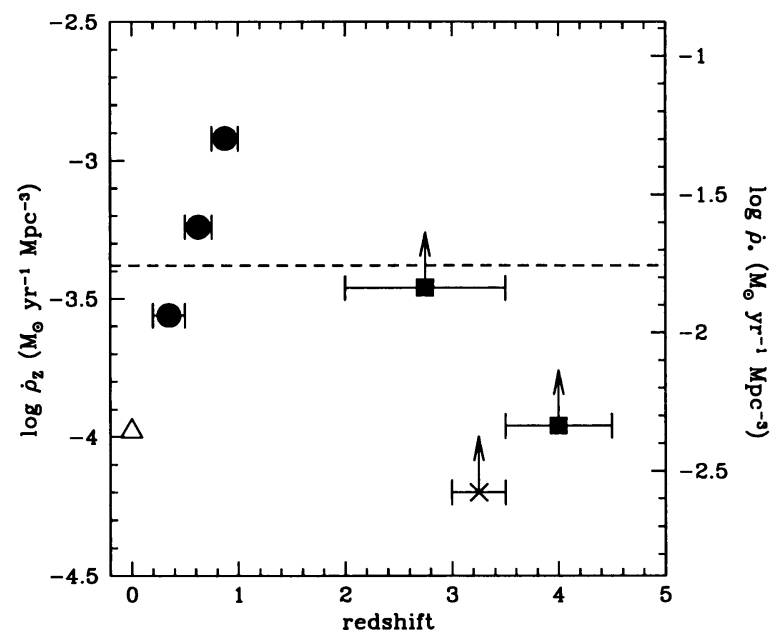

Figure 1. Star-formation density limits from the compilation of [6]. The points are: triangles - local $\mathrm{H} \alpha$ survey [2], filled dots - the CFRS survey[5], diagonal cross - lower limits from Lyman limit galaxies observed by [7], filled squares from $\mathrm{HDF}[6]$. The dashed line shows the average rate required to produce the current abundance of metals for $\Omega=1$ and $H_{0}=50 \mathrm{~km} \mathrm{~s}^{-1} \mathrm{Mpc}^{-1}$.

\section{The WHT/TTF survey}

The first survey to be presented here is a narrow-band imaging survey for emission line objects in the Hubble Deep Field. This is unique in our use of the 'TAURUS Tunable Filter' [1] to image very narrow (10§) slices. Unlike standard narrow-band glass filters the narrower, wavelength tunable, bandwidth is perfectly matched to the typical linewidths $\left(\sim 300 \mathrm{~km} \mathrm{~s}^{-1}\right)$ of normal galaxies. TTF uses Fabry-Perot technology: the TTF etilon has an unusually small band-gap - between $2-15 \mu \mathrm{m}$ which gives $\Delta \lambda=10$ $60 \AA$ (c.f. $100-200 \mu \mathrm{m}$ and $\Delta \lambda=1-2 \AA$ for a conventional FP). In our HDF survey at the William Herschel Telescope in La Palma we scan for [OII] emission over $0.9<z<1.5$ in $3 \mathrm{OH}$-line free regions of the $I$-band: I1: $z=0.894-0.908(0.2 \mathrm{hr} /$ slice $), \mathrm{I} 5: z=1.173-1.191(1 \mathrm{hr} /$ slice $)$ and I8: $z=1.426-1.448,(1.5 \mathrm{hr} / \mathrm{slice})$. We cover a volume of $\sim 3000 \mathrm{Mpc}^{3}$ down to fluxes of $10^{-21} \mathrm{~W} \mathrm{~m}^{-2}$ which corresponds to SFRs as low as $1-2 M_{\odot} \mathrm{yr}^{-1}$ at these redshifts. While we are still analyzing this data our initial work shows emission line objects appearing at about the rate expected from an extrapolation of Figure 1. Some examples are shown in Figure 2. 
Figure 2. Candidate emission line objects (indicated by circles) at different redshifts in the Hubble Deep Field). These images show two slices at different wavelengths which have been continuum subtracted (removing over $98 \%$ of the objects).

\section{CGS4 Observations of $\mathrm{H} \alpha$ in known $z \sim 1$ galaxies}

A related project we are pursuing is to try and detect the $\mathrm{H} \alpha$ line in a sample of known $z \sim 1$ normal field galaxies from the CFRS/LDSS2 samples ([4], [3]). At this redshift the line is in the $J$-band. Our strategy is to observe at high spectral resolution $(R>2000)$ and select from the 74 galaxies with $0.9<z<1.4$ those with redshifted $\mathrm{H} \alpha$ lying in regions between the night sky $\mathrm{OH}$ lines - this greatly reduces our effective background and lets us reach fluxes of $10^{-19} \mathrm{~W} \mathrm{~m}^{-2}$ in just a few hours.

By measuring the $\mathrm{H} \alpha$ line we get a independent, well-calibrated measure of the SFR in these systems which we can compare with the UV-derived measurements of [5]. Moreover at these redder wavelengths extinction is less and comparison can address the big question of the importance of dust in these objects. This sample has already been subject to a great deal of morphological analysis from $H S T$ data so we can also look at the SFR in different systems, and correlations with parameters such as asymmetry and bimodality (candidate mergers).

In our survey so far (using CGS4 on the UK Infrared Telescope in Hawaii) we have observed 13 galaxies and reliably detected $\mathrm{H} \alpha$ in 8 of these. Most of the galaxies also have the continuum detected in $J$ which indicates reliable object acquisition. From the $\mathrm{H} \alpha$ fluxes we can make a first pass at estimating the SFR - this is shown in Figure 3. Our preliminary, tentative, conclusion is that we find none of these $z \sim 1$ galaxies have greatly 

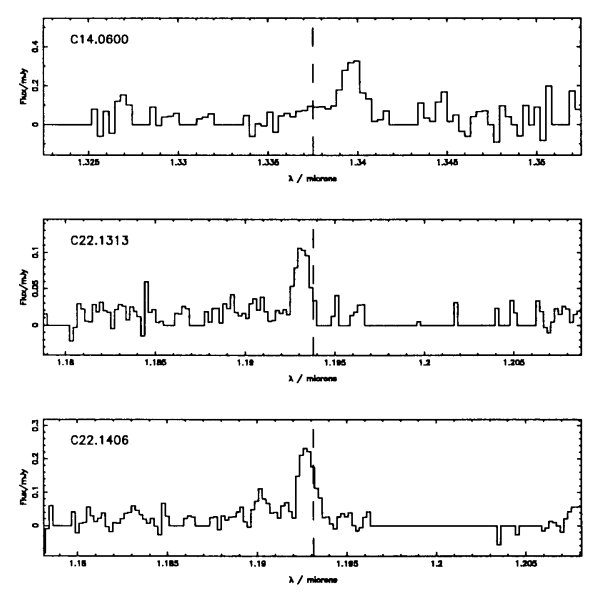

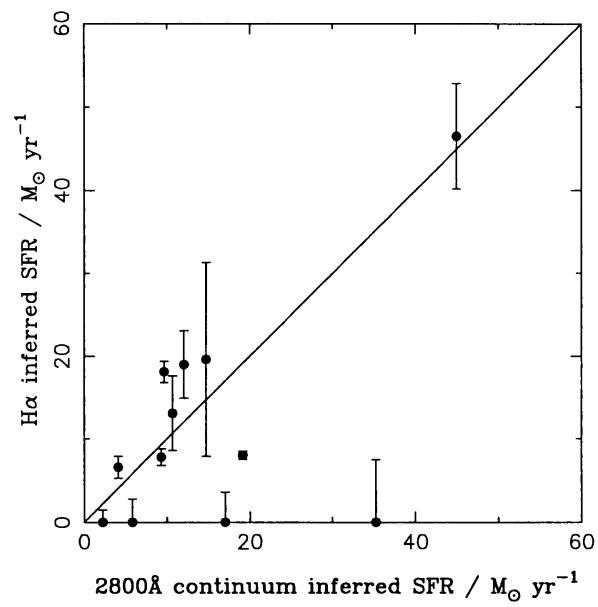

Figure 3. Left: some sample $\mathrm{H} \alpha$ spectra. Right: SFR per object as inferred from $\mathrm{H} \alpha$ and UV indices.

enhanced SFR from H $\alpha$ c.f. UV estimates. Rather we find good agreement. This suggests to us that dust extinction is not of major importance in this sample of objects (though of course we can not rule out hidden, 'dark galaxies'), confirming the Madau et al point at $z \sim 1$. We do note that some galaxies show UV but not $\mathrm{H} \alpha$ flux. Probably the most likely explanation is the differing lifetimes of young stars contributing at $2800 \AA\left(\sim 10^{9} \mathrm{yr}\right)$ and $\mathrm{H} \alpha\left(\sim 10^{7} \mathrm{yr}\right)$, or perhaps some of the UV flux does not come from hot young main sequence stars (e.g. AGN). We are currently investigating this further by means of comparing SED fits to populations vs $\mathrm{H} \alpha$ strength.

In conclusion we observe that the detection of emission lines in normal galaxies with modest SFRs is now possible at $z>1$, even with $4 \mathrm{~m}$ telescopes. The technique of $\mathrm{OH}$ avoidance augurs well for future near-IR studies of normal galaxies at high redshift.

\section{References}

1. Bland-Hawthorn J., Jones D. H.,1997, MNRAS, in preparation (astroph/9707315).

2. Gallego J., Zamorano J., Arágon-Salamanca A., Regg M., 1995, ApJ, 455, L1

3. Glazebrook K., Ellis R. S., Colless M. M, Broadhurst T. J., Allington-Smith J. R., Tanvir N. R., 1995A, MNRAS, 273, 157

4. Lilly S. J., Le Fevre O., Crampton. D., Hammer F.,Tresse L., 1995, ApJ, 455, 50

5. Lilly S. J., Le Fevre O., Hammer F., Crampton. D., 1996, ApJ, 460, L1

6. Madau P., Ferguson H. C., Dickinson M. E., Giavalisco M., Steidel C. C., Fruchter A., 1996, MNRAS, 283, 1388

7. Steidel C. C., Giavalisco M., Dickinson M. E., Adelberger K. L., 1996, AJ, 112, 352 